\title{
Design, Control, and Experimental Performance of a Teleoperated Robotic Fish
}

\author{
Evangelos Papadopoulos, Senior Member IEEE, Efthymios Apostolopoulos and Petros Tsigkourakos \\ Department of Mechanical Engineering \\ National Technical University of Athens \\ 15780 Athens, Greece \\ egpapado@central.ntua.gr
}

\begin{abstract}
Fish-like propulsion is a challenging alternative to propellers in small underwater vehicles. This paper presents the analysis, the design stages, the development and the experimental evaluation of a small low-cost teleoperated underwater robotic fish, driven by an oscillating foil. The main principles for the development of efficient thrust by oscillating foils are presented, and implemented. Essential mechatronic subsystems of the robot, including the tail's motion control system, the wireless communication system, and the autonomous power system are described. Design equations are provided, evaluation experiments are executed and performance results are presented.
\end{abstract}

Keywords-Fish propulsion, robotic fish design, oscillating foil, underwater robot analysis

\section{INTRODUCTION}

In recent years, the Underwater Unmanned Vehicles (UUVs) capabilities required to complete underwater missions have become more demanding. A UUV must be capable of traveling at high speeds, turning and maneuvering in tight spaces using few and efficient actuators. The most common UUVs use propellers for their propulsion, and lifting surfaces for maneuvering. These vehicles exhibit poor performance and limited maneuverability. Their small propellers have a maximum reported efficiency that does not exceed $40 \%$ while the required radius for a $180^{\circ}$ turn is equal to several vehicle lengths [1]. This kind of propulsion has matured, leading to the research of alternative propulsion systems.

Fish and marine mammals provide a great paradigm of efficient thrust generation since they employ mechanisms that allow them to swim very fast, accelerate rapidly from rest, and perform a 180 degree turn within a fraction of their body length. Due to the fact that many fish tails resemble high aspect ratio foils, a number of studies on oscillating foils have been carried out to investigate fish locomotion principles. Triantafyllou et al. proved experimentally that, under specific conditions, an oscillating foil could produce significant thrust, accompanied by high efficiency due to the formation of a reverse Karman vortex street [2]. Based on their experimental results, oscillating foils have been proposed as an alternative propulsion system. Their good performance combined with their stealth operation, made their implementation in small underwater vehicles very promising. Additionally, the development of the MIT
Robotuna, a fish-like robotic mechanism, showed that a streamlined actively swimming body requires less power, than a streamlined rigid body of the same size and speed [3]. Besides Robotuna, MIT's Robopike and Draper's VCUUV are also well known examples of fish-like robots [4], [5].

This paper focuses on the analysis, the design and the development of a low-cost, small size, robotic fish that is propelled by an oscillating foil. The main principles for efficient thrust generation by oscillating foils are presented. Essential subsystems of the vehicle, including the tail motion control system, the wireless communication system and the autonomous power system are described in detail. The vehicle is characterized by (a) an oscillating foil propulsion system, (b) power autonomy of at least one hour, (c) untethered movement across rectilinear and curviform trajectories (2-D motion). Evaluation experiments are executed and performance results are presented and discussed. Useful conclusions concerning the hydrodynamic mechanism of propulsion and the related design equations are provided.

\section{RoBOTIC FISH PROPULSION AND DYNAMICS}

Main fish swimming locomotion modes include anguilliform, carangiform, and ostraciiform, depending on the body percentage that contributes to thrust [6]. These result in three alternative mechanical concepts (Fig.1):

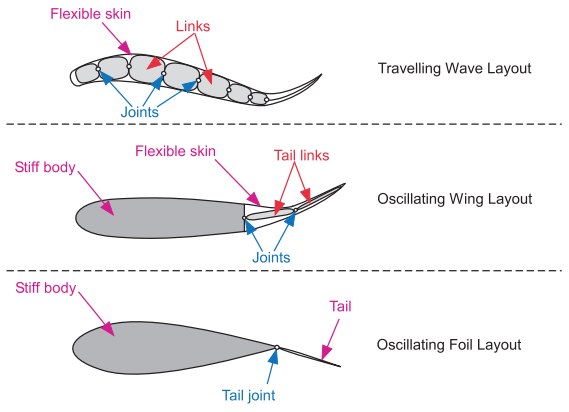

Figure 1. Alternative ways for robot fish design, adapted from [10].

(a) Traveling wave: Most of the body contributes to thrust production via undulatory motion. Design of extremely small robots using Shape Memory Alloy (SMA) wires is possible but their poor efficiency and the many required joints that increase friction losses render the concept unsuitable. 
(b) Oscillating wing: The rear third of the body, comprised by the tail and the caudal fin, contributes to thrust production. This design imitates the most efficient fishswimming mode, however, it is still relatively complex for very small vehicles.

(c) Oscillating plate: Only the tail contributes to thrust production through oscillatory motion. Hydrodynamically, this design is the least efficient due to the rigid body. However, it provides the simplest design: fewer joints, less mechanical losses due to friction, simple and inexpensive construction, simple control and less weight. Therefore, concept (c) was selected.

\section{A. Thrust Generation in Oscillating Foils}

Oscillating foils (heaving and/or pitching) produce thrust through the formation of an unstable jet flow, known as the reverse Karman vortex street. According to experimental results, the parameters that affect the thrust generation and the efficiency of an oscillating foil are: (a) the shape of the foil, (b) the non-dimensional Strouhal number St, (c) the maximum angle of attack $\alpha_{\max }$, (d) the heave motion amplitude $h_{o}$ to chord $c$ ratio, $h_{o} / c$, and (e) the pitch to heave phase angle $\psi[2,7]$. Fig. 2 shows the basic oscillating foil parameters.

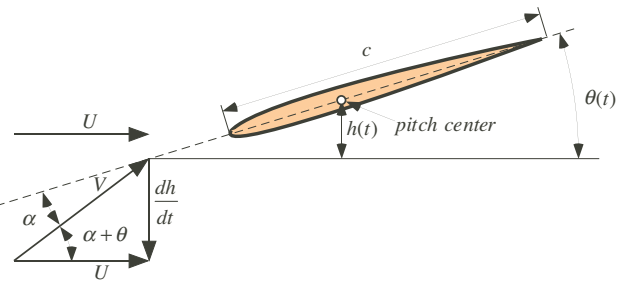

Figure 2. Parameters affecting the motion of an oscillating foil.

The Strouhal number is defined as

$$
S t=f \cdot d / U
$$

where $f$ is the frequency of the foil's oscillation, $d$ is the width of the wake (maximum travel of the trailing edge) and $U$ is the forward speed of the vehicle. Significant thrust accompanied by high efficiency can be obtained for $S t$ numbers between 0.25 and 0.45 and for angles of attack between $15^{\circ}$ and $25^{\circ}$ [7]. Depending on the fish propulsion mode, some, or all of the aforementioned parameters, are taken into consideration during the design process. Since the oscillating plate concept, characterized by the foil's pitching motion, is employed, it allows us to neglect the parameters of heave-to-chord ratio and pitch-to-heave phase angle.

\section{B. Robotic Fish Dynamics and Design Equations}

The robotic fish is divided into the main body and the tail. Based on fish observations, the main body is modeled as a 3D ellipsoid with length $L_{1}$, height $L_{2}$, width $L_{3}$ and ratios $L_{2}=0.5 L_{1}$ and $L_{3}=0.3 L_{1}$. The tail is modeled as a rectangular with chord length $H_{1}=\mathrm{c}$, height $\mathrm{H}_{2}$, width $\mathrm{H}_{3}$ and ratio $H_{2}=2 H_{1}$. The tail executes a sinusoidal motion described by

$$
\theta(t)=\theta_{a}+\theta_{0} \cdot \cos (2 \pi f t)
$$

where $\theta(t)$ is the angle between the tail and the longitudinal axis of the vehicle, $\theta_{a}$ is the mean value of $\theta$ during one period, $\theta_{0}$ is the pitch amplitude and $f$ is the frequency of the tail's oscillation.

The forces that act on the main body when it travels along a straight path include the inertial force $F_{\text {inertial }}$, and the hydrodynamic drag $F_{D}$. The sum of these two forces equals to the mean propulsive force $F_{T}$ and they are given by

$$
\begin{gathered}
F_{\text {inertial }}=\left(m_{\text {vehicle }}+m_{\text {added }}\right) \cdot \dot{U} \\
F_{D}=0.5 \cdot \rho_{w} \cdot c_{D} \cdot A \cdot U^{2}
\end{gathered}
$$

where $m_{\text {vehicle }}$ is the mass of the body, $m_{\text {added }}$ is the added mass of the accelerated water around the vehicle (estimated using tables in [8]), $\rho_{w}$ is the density of the water, $A$ is the frontal area of the vehicle, and $c_{D}$ is the drag coefficient which depends on the shape of the body and the Reynolds number. Setting the vehicle's length and speed to $L_{l}=30 \mathrm{~cm}$ and $U=30 \mathrm{~cm} / \mathrm{s}$ respectively, the maximum expected Reynolds number is $R e=9 \cdot 10^{4}$. Therefore, the flow around the body is expected to be laminar. According to [9], for rotationally symmetrical bodies and laminar flows, the coefficient $c_{D}$ is given by

$$
c_{D}=0.33 \cdot(d / l)+c_{\text {flam }} \cdot\left[3 \cdot(l / d)+3 \cdot(d / l)^{1 / 2}\right]
$$

where $d$ is the maximum diameter of the body, $l$ is the length of the body and $c_{\text {flam }}$ is the skin friction drag coefficient in laminar flow (given in Fig. 6-2 in [9]). According to (5), for $l / d=2.5$ and $c_{\text {flam }}=0.015$, the coefficient $c_{D}$ equals to 0.27 .

In tail oscillation, thrust production results from the acceleration of the water in the vicinity of the tail. Assuming that the accelerated water is contained in a cylinder enclosing the tail, with radius $H_{2} / 2$ and length $H_{1}$ the required motor torque is given by

$$
T_{\text {added }}=I_{z z}^{a m} \dot{\omega}_{L}=\rho_{w} \frac{\pi}{2} \int_{0}^{H_{1}} y^{2}\left[2\left(H_{2} / 2\right)^{2}\right] d y \dot{\omega}_{L}=261.27 H_{2}^{2} H_{1}^{3} \dot{\omega}_{L}
$$

where $I_{z z}^{a m}$ is the polar moment of inertia of the accelerated mass of water and $\dot{\omega}_{L}$ is the angular acceleration of the tail. Although this assumption looks reasonable (though arbitrary to some extent) the only way to know the exact $I_{z z}^{a m}$ is through experiments which will be discussed in section IV. The tail inertia is much smaller than that of the water and is neglected here. The associated power is

$$
P_{\text {added }}=T_{\text {added }} \cdot \omega_{L}
$$

where $\omega_{L}$ is the tail angular speed. For the case of sinusoidal motion, the average propulsive power is computed by averaging the term $P_{\text {added }}$ over one period of oscillation $T=1 / f$. Since only a percentage of the power, used for water acceleration, is transformed into propulsive power, the result is multiplied by a factor $\eta$ assumed to be around 0.5 .

$$
P_{T}=\eta \cdot \bar{P}_{\text {added }}=\eta \cdot 8 \cdot I_{z z}^{a m} \cdot f^{3} \cdot \pi^{2} \cdot \theta_{0}^{2}
$$


For constant cruising speed $U$, the power dissipation $P_{D}$ due to the hydrodynamic drag equals to the average propulsive power $P_{T}$. Combining (1), (4) and (8) results in

$$
\frac{L_{1}}{H_{2}}=6.51 \cdot \sqrt{\frac{\eta \cdot S t^{3} \cdot \theta_{0}{ }^{2}}{\sin \left(\theta_{0}\right)^{3} \cdot c_{D}}}
$$

According to (9), for a body length $L_{1}=30 \mathrm{~cm}, S t$ in the range of $[0.25-0.45], C_{D}=0.27$ and $\theta_{0}=15^{\circ}$, the tail's dimensions vary from $H_{2}=13.6 \mathrm{~cm}$ and $H_{1}=6.8 \mathrm{~cm}$ to $H_{2}=5.6$ $\mathrm{cm}$ and $H_{1}=2.8 \mathrm{~cm}$. From the above range, we chose $H_{2}$ and $H_{l}$ to be $13.4 \mathrm{~cm}$ and $6.7 \mathrm{~cm}$, respectively. The resulting inertia of the displaced water is $I_{z z}^{a m}=0.0013 \mathrm{~kg} \cdot \mathrm{m}^{2}$.

However, according to [6], high aspect ratio lunateshaped tails exhibit improved hydrodynamic efficiency compared to rectangular foils of the same overall dimensions because they induce less drag per unit of generated thrust and therefore a lunate-shaped tail with $H_{2}=13.4 \mathrm{~cm}$ and $H_{1}=6.7 \mathrm{~cm}$ was implemented. For a given body length, tail size, pitch amplitude, and vehicle desired speed $U,(4)$ and (8) yield

$$
f=1.85 \cdot \sqrt[3]{\frac{c_{D} \cdot A \cdot U^{3}}{\eta \cdot I_{z z}^{a m} \cdot \theta_{0}^{2}}}=0.142 \cdot \sqrt[3]{\frac{c_{D} \cdot L_{1}^{2} \cdot U^{3}}{\eta \cdot H_{2}^{2} \cdot H_{1}^{3} \cdot \theta_{0}^{2}}}
$$

As an example, the aforementioned tail must oscillate at $2.23 \mathrm{~Hz}$ to achieve a speed of $30 \mathrm{~cm} / \mathrm{s}$. For a more detailed calculation of the tail's torque load, the torque due to drag $T_{\text {damp }},[10]$, and due to tail acceleration $T_{\text {inertial }}$, must be added to $T_{\text {added }}$. These are given by

$$
\begin{gathered}
T_{\text {damp }}=0.5 \cdot \rho_{w} \cdot c_{D t} \cdot H_{2} \cdot \omega_{L} \cdot\left|\omega_{L}\right| \cdot \int_{0}^{H_{1}} r^{3} d r \\
T_{\text {inertial }}=I_{\text {inertial }} \dot{\omega}_{L}=\rho_{f} H_{1} H_{2} H_{3}\left(4 H_{1}^{2}+H_{3}^{2}\right) / 12 \cdot \dot{\omega}_{L}
\end{gathered}
$$

where $I_{\text {nertial }}$ is the polar moment of inertia of the tail, and $\rho_{f}$ is the mass density of the tail. The total torque is given by

$$
T_{L}=T_{\text {added }}+T_{\text {damp }}+T_{\text {inertial }}
$$

\section{DESIGN AND IMPLEMENTATION}

In this section the robotic fish design is described. During the design process, a number of decisions were taken including the selection and the sizing of the tail's actuator, the motion control system, the wireless communication and the power autonomy of the vehicle.

\section{A. Tail Actuator}

In order to have the underwater vehicle capable of swimming at various speeds, turning and maneuvering, the tail motion system should be able to control $f, \theta_{\alpha}$ and $\theta_{0}$ in (2). Three designs with their associated actuators were thus studied.

(a) Two antagonistic linear actuators: SMA wires can be used providing simplicity and symmetry in the system's dynamics. However, their poor efficiency (3\%) and the need for a distinct drive for each actuator made this design inappropriate.

(b) An actuator and an antagonistic spring: SMA wires or piezoelectric (PZT) actuators can be employed. It requires double actuator displacement and exhibits asymmetry in the dynamics. SMA wires present the same problems as above, while the PZT require a wide voltage range and expensive transmissions. Therefore, this design was also rejected.

(c) An actuator that provides alternating displacements: This design can be implemented using voice coils, stepper motors or DC motors. The actuator's displacement is converted into tail motion by a simple transmission. This design has the advantage of a single actuator drive. The increased power consumption due to alternating accelerations/decelerations of the actuator inertia is the only drawback. Among the three candidate actuators, DC motors were considered suitable because they can be combined with a gearhead and encoder in a single package, minimizing the weight/power and simplifying the design and the control.

The DC motor was sized for the most demanding load at which the vehicle travels at constant speed $U=30 \mathrm{~cm} / \mathrm{s}$ with $f=2.5 \mathrm{~Hz}$ and $\theta_{0}=15^{\circ}$. The tail load $T_{L}$ and the angular speed $\omega_{L}$ were calculated using (2), (6), (11), (12) and (13). The $\mathrm{DC}$ motor that satisfied the requirements is the $1.5 \mathrm{~W}$ RE10 from Maxon Motors, with an integrated GP10K gearhead (reduction 64:1) and a digital MR encoder (16 CPT, 2 ch) in a single unit of $d=10 \mathrm{~mm}, l=48.4 \mathrm{~mm}$ and $m=17 \mathrm{gr}$.

\section{B. Motion Control System}

In order to get the desired tail motion, described by (2), a closed loop control system for the position $\theta(t)$ of the tail was implemented. Since the direct measurement of $\theta$ would complicate the design, the motor shaft position $\theta_{m}$ was measured using the encoder. The elements that realize the control system are shown in Fig. 3 and include:

(a) A Microchip PIC16F876 microcontroller $(\mu C)$ which reads the reference angle $\theta_{m R}$, implements the discrete control law and generates a PWM control signal for the motor driver.

(b) An Intersil 500mA full-bridge MOSFET power driver HIP4020, which is easily interfaced with $\mu \mathrm{Cs}$.

(c) An Agilent quadrature decoder/counter HCTL2016 that interfaces the encoder signal to the $\mu \mathrm{C}$, quadruplicates the encoder resolution, and rejects noise.

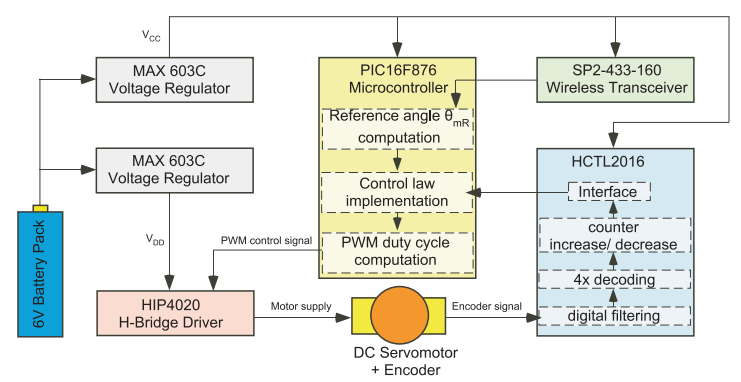

Figure 3. Schematic diagram of the tail motion control system. 
The PCB that realizes the control loop, running at $200 \mathrm{~Hz}$, is shown in Fig. 4. At each loop the $\mu \mathrm{C}$ reads the motor angle $\theta_{\mathrm{m}}[k]$ from the HCTL2016, calculates the control voltage $V[k]$ and generates the PWM signal. In order to calculate the voltage command $V[k]$, the PIC implements the law

$$
V[k]=b \cdot e[k-1]+c \cdot e[k]
$$

where $e[k]=\theta_{m R}[k]-\theta_{m}[k]$. The discrete controller gains $b$, and $c$ are found by emulating a continuous time PD controller designed for sizing the motor. The value of $V[k]$ is translated by the $\mu \mathrm{C}$ into an appropriate PWM duty cycle which is sent to HIP4020 along with a direction signal that depends on the sign of $V[k]$. The $\theta_{m R}[k]$ computation is accomplished by discrete sampling of 128 values located in the PIC's EEPROM that describe one quarter of a sinus. The variation of the tail frequency $f$ is implemented by changing the sampling period $\mathrm{N}$. The change in amplitude $\theta_{0}$ is obtained by multiplying the data with an appropriate factor, whereas the variation of $\theta_{a}$ is obtained by adding the desirable angle.

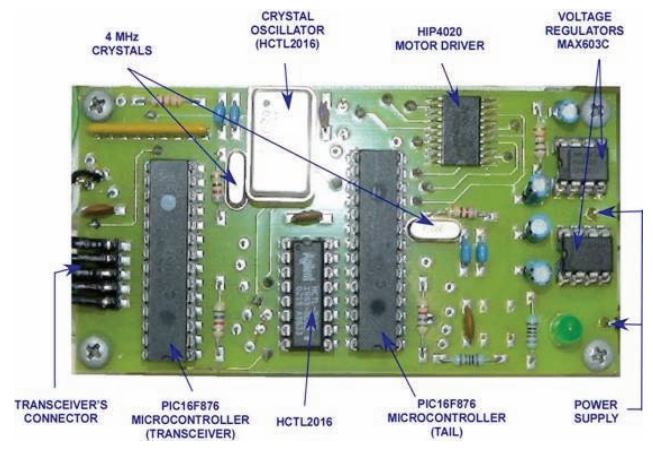

Figure 4. Control system PCB.

\section{Wireles Communication}

The motion parameters $f, \theta_{\alpha}$ and $\theta_{0}$, which determine the vehicle's speed and orientation, are transmitted from the operator's PC to the tail motion control system through a wireless communication link. To establish this link two Radiometrix SP2-433-160 transceiver modules were selected. They combine a UHF radio transceiver and a 160 kbps fast radio packet controller. Each module is controlled by a PIC16F876 host $\mu \mathrm{C}$, accompanied by a $433 \mathrm{MHz}$ whip antenna. The whole setup is implemented in two PCBs. One is placed inside the vehicle and the other is connected to the operator's PC. The communication is supported by a GUI developed in LabVIEW that allows the user to control the speed and the direction of the vehicle.

\section{Power System \& Hull Design}

In order to provide the vehicle with power autonomy for at least one hour the total current consumption from the ICs, the motor, the RF transceiver and the CMOS sensor was measured and a battery pack of five AAA NiMH cells, each having $550 \mathrm{mAh}$ capacity, was selected. Additionally, two Maxim MAX603C $500 \mathrm{~mA}$ linear voltage regulators provide $5 \mathrm{~V}$ fixed supply to the motor driver IC and to the rest of the ICs separately so as to isolate the electric noise produced by the motor driver.
All the subsystems are integrated in a $33.7 \mathrm{~cm}$ long robotic fish which consists of a $27 \mathrm{~cm}$ long body and a 6.7 $\mathrm{cm}$ long moon shaped tail, connected through a revolute joint. Since the motor was placed away from the tail shaft, an assembly of pulleys and cables was implemented in order to transmit the motion to the tail The body is rigid and divided into two main parts: The inner hull houses and seals all the electronic components while the outer hull is free flooded and provides a streamlined shape, (Fig. 5).
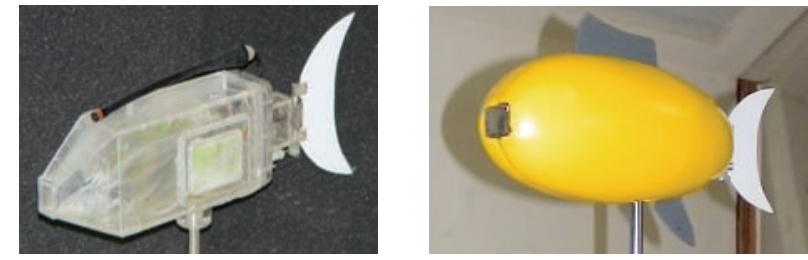

Figure 5. Inner hull from Plexiglas (left) and outer hull from foam (right).

\section{EXPERIMENTAL RESULTS}

In order to evaluate the design methodology a number of experiments were conducted. The tail motion system was evaluated, the vehicle's hydrodynamic drag was measured and the thrust produced by the oscillating tail was estimated.

\section{A. Tail Motion System}

To identify possible discrepancies in the design methodology, the tail motion in the water was recorded and compared to the response predicted by simulation. To this end, the vehicle was immersed in a cylindrical water tank and was rigidly attached to a stationary platform placed over the tank. During the experiments, the moon-like shaped tail $\left(H_{1}=6.7 \mathrm{~cm}, H_{2}=13.4 \mathrm{~cm}\right)$ executed the motion described by (2). Frequency f ranged from 0.5376 to $3.125 \mathrm{~Hz}$, while $\theta_{0}$ and $\theta_{\alpha}$ remained constant at $15^{\circ}$ and $0^{\circ}$, respectively. The tail angle was provided by the HCTL2016 and the data were transferred through the wireless communication to the PC.

The associated simulation model comprises the discrete controller, the PWM signal and the plant model. The control system is hybrid since the controller is modeled in discrete time and the plant is modeled in continuous time. The plant model consists of the motor-gearhead pack ( $R$ and $L$ of the windings, torque constant $K_{\mathrm{T}}$ and reduction 64:1), the tail (hydrodynamic drag and equivalent inertia $I_{z z}$ ) and friction. Friction is modeled using the GKF (General Kinetic Friction) model [11]:

$$
T_{f}\left(\omega_{L}\right)=\left[T_{c}+\left(T_{s}-T_{c}\right) \exp \left(-\left|\omega_{L} / \omega_{s t r}\right|^{2}\right)\right] \cdot \operatorname{sgn}\left(\omega_{L}\right)+b \omega_{L}
$$

where $T_{s}$ is the breakaway torque, $T_{c}$ is the Coulomb friction level and $b$ is the viscous friction coefficient. The friction parameters were identified experimentally [11].

Fig. 6a presents the experimental and the simulated tail motion. At low frequencies the responses are identical while at high frequencies a deviation exists, which increases with frequency. By examining the simulation model, it was concluded that this discrepancy was due to the tail equivalent inertia and especially to the added mass inertia. To be able to match the data, the equivalent inertia was reduced by a factor 
of nineteen. To some extent this was expected, as the water inertia in (6) was very approximate. With this adjustment, experiments and simulation match well, (Fig. 6b).
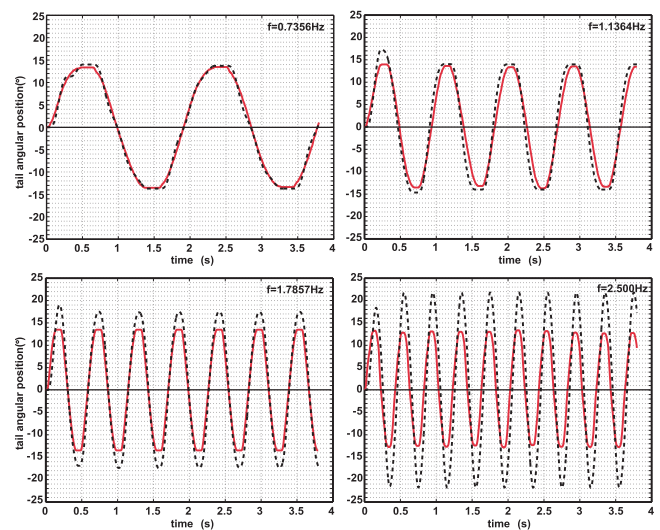

(a)
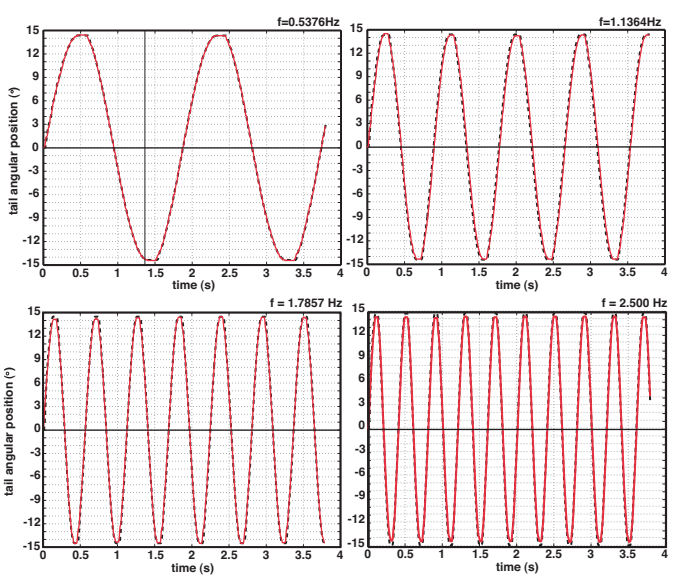

(b)

Figure 6. Tail motion as a function of time for various frequencies: ---experiment; - - - simulation (a) initial simulation model, (b) improved simulation model.

\section{B. Hydrodynamic Drag}

To identify the vehicle's hydrodynamic drag and compare it to the theoretical model, the vehicle was placed inside a wind tunnel and an ATI Nano17 force/torque sensor was attached to it in order to provide drag measurements. Experimental data were collected to a PC through a NI DAQ card. The vehicle's aerodynamic drag was measured for air speeds between 0.1 and $10 \mathrm{~m} / \mathrm{s}$ (Fig.7a). An error tolerance of $0.003 \mathrm{~N}$ was added due to sensor noise. Using dimensional analysis and the Buckingham Pi theorem, the hydrodynamic drag of the robotic fish is obtained. This is done by equating the drag coefficient and the Reynolds number, which are non-dimensional.

$$
\begin{aligned}
& c_{D, a i r}=c_{D, \text { water }} \Rightarrow \frac{F_{D, \text { air }}}{\rho_{\text {air }} \cdot U_{\text {air }}^{2}}=\frac{F_{D, \text { water }}}{\rho_{\text {water }} \cdot U_{\text {water }}^{2}} \\
& \operatorname{Re}_{\text {air }}=\operatorname{Re}_{\text {water }} \Rightarrow \frac{\rho_{\text {air }} \cdot U_{\text {air }}}{\mu_{\text {air }}}=\frac{\rho_{\text {water }} \cdot U_{\text {water }}}{\mu_{\text {water }}}
\end{aligned}
$$

Fig. $7 \mathrm{~b}$ shows the experimental hydrodynamic drag and that computed by (4). The theoretical drag lacks slightly compared to the experimental one due to the fact that (4) is referred to smooth streamlined bodies while the vehicle's body has a number of surface imperfections. Additionally, (4) does not take into account the drag that is added to body by the tail and the fins. This deviation can be eliminated by multiplying the drag coefficient in (5) with a compensating factor of 1.2 yielding a very good approximation of the vehicle's drag.

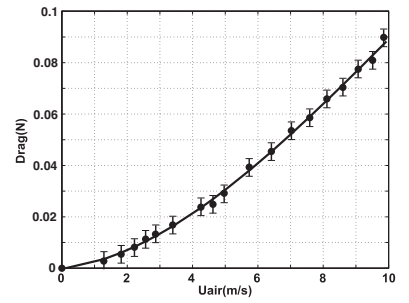

(a)

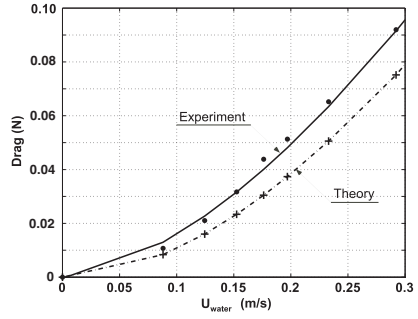

(b)
Figure 7. (a) Vehicle's aerodynamic drag as a function of air speed, (b) Experimental and theoretical vehicle's hydrodynamic drag.

\section{Velocity and Thrust Measurements}

Since the direct measurement of the thrust produced by the tail was not feasible with our equipment, an indirect method was used. When the vehicle travels at constant speed, drag equals to thrust. Knowing from Fig. $7 \mathrm{~b}$ the vehicle's drag as a function of speed, the thrust can be estimated using speed measurements of the robotic fish for a given tail frequency. For these experiments, a water tank was used, along which a tape measure was attached. The vehicle was traveling along the tank at a constant speed and every 30 $\mathrm{cm}$ the time was recorded using a digital timer. Tail frequency f ranged from 0.5376 to $3.125 \mathrm{~Hz}$, while $\theta_{0}$ remained at $15^{\circ}$.

Table I compares the experimental vehicle speed, $U_{\text {exp }}$, to the theoretical one, $U_{\text {theor }}$, i.e. the speed calculated using (4), (8) and the fact that for constant speed $F_{T}=F_{D}$. A significant difference between $U_{\text {exp }}$ and $U_{\text {theor }}$ can be observed. This is due to the fact that $I_{z z}^{a m}$ in (6) is very approximate (calculated here to be $13010^{-5} \mathrm{kgm}^{2}$ ) while the experimental hydrodynamic drag is slightly larger than the theoretical one. In order to validate this assumption, $I_{z z}^{a m}$ was estimated experimentally using $f-U_{\text {exp }}$ pairs in Table I, and experimental drag data in Fig. $7 \mathrm{~b}$ and (8). Table II displays $I_{z z, \exp }^{a m}$ and the ratio $I_{z z}^{a m} / I_{z z, \exp }^{a m}$. The average $I_{z z, \exp }^{a m}$ was found to be $6.77 \cdot 10^{-5} \mathrm{~kg} \mathrm{~m}^{2}$, i.e. nineteen times less than the calculated one. This is in agreement with the results presented in section IV-A. To improve the initial design equations a factor $k=1 / 19$ was added to (6) resulting in

$$
I_{z z, a d j}^{a m}=k \cdot 261.27 \cdot H_{2}^{2} \cdot H_{1}^{3}=13.75 \cdot H_{2}^{2} \cdot H_{1}^{3}
$$

The corrected design equations (9) and (10) become 


$$
\begin{gathered}
\frac{L_{1}}{H_{2}}=1.514 \cdot \sqrt{\frac{\eta \cdot S t^{3} \cdot \theta_{0}{ }^{2}}{\sin \left(\theta_{0}\right)^{3} \cdot c_{D}^{\prime}}} \\
f=4.93 \cdot \sqrt[3]{\frac{c_{D}^{\prime} \cdot A \cdot U^{3}}{\eta \cdot I_{z z}^{a m} \cdot \theta_{0}^{2}}}=0.378 \cdot \sqrt[3]{\frac{c_{D}^{\prime} \cdot L_{1}^{2} \cdot U^{3}}{\eta \cdot H_{2}^{2} \cdot H_{1}^{3} \cdot \theta_{0}^{2}}}
\end{gathered}
$$

with $c_{D}^{\prime}=1.2 c_{D}$. To validate the corrected design equations, a new larger tail was used with $H_{2}=15.2 \mathrm{~cm}$. From (19) the tail frequency required for a speed $U=30 \mathrm{~cm} / \mathrm{s}$ was $4.62 \mathrm{~Hz}$. To avoid overloading of the servomotor, the experiments were conducted with tail frequencies up to $3.125 \mathrm{~Hz}$.

TABLE I. $\quad U_{\text {VEHILLE }}$ (EXPERIMENT VS. THEORY)

\begin{tabular}{|c|c|c|}
\hline$f[\mathrm{~Hz}]$ & $U_{\text {exp }}[\mathrm{m} / \mathrm{s}]$ & $U_{\text {theor }}[\mathrm{m} / \mathrm{s}]$ \\
\hline 0.5376 & 0.018 & 0.095 \\
\hline 0.8929 & 0.028 & 0.129 \\
\hline 1.1364 & 0.043 & 0.163 \\
\hline 1.3889 & 0.058 & 0.200 \\
\hline 1.7857 & 0.081 & 0.257 \\
\hline 2.0833 & 0.085 & 0.300 \\
\hline 2.5000 & 0.121 & 0.360 \\
\hline 3.1250 & 0.157 & 0.450 \\
\hline
\end{tabular}

TABLE II. EXPERIMENTAL CALCULATION OF $I_{z z}^{a m}$

\begin{tabular}{|c|c|c|c|c|}
\hline$f[\mathrm{~Hz}]$ & $U_{\exp }[\mathrm{m} / \mathrm{s}]$ & $F_{\mathrm{D}}(\mathrm{N})$ & $I_{z z, \exp }^{a m}\left[\mathrm{kgm}^{2}\right]$ & $I_{z z, t h}^{a m} / I_{z z, \exp }^{a m}$ \\
\hline 0.5376 & 0.018 & 0.002 & $8.63 \mathrm{E}-05$ & 15,06 \\
\hline 0.8929 & 0.028 & 0.004 & $5.86 \mathrm{E}-05$ & 22,18 \\
\hline 1.1364 & 0.043 & 0.006 & $6.55 \mathrm{E}-05$ & 19,85 \\
\hline 1.3889 & 0.058 & 0.009 & $7.26 \mathrm{E}-05$ & 17,92 \\
\hline 1.7857 & 0.081 & 0.013 & $6.89 \mathrm{E}-05$ & 18,87 \\
\hline 2.0833 & 0.085 & 0.014 & $5.64 \mathrm{E}-05$ & 23,05 \\
\hline 2.5000 & 0.121 & 0.023 & $6.64 \mathrm{E}-05$ & 19,59 \\
\hline 3.1250 & 0.157 & 0.035 & $6.71 \mathrm{E}-05$ & 19,38 \\
\hline
\end{tabular}

Table III compares the experimental speed $U_{\text {exp }}$, to the corrected theoretical one $U_{\text {theor }}$, as computed using (4), (8), (17) and $c_{D}^{\prime}=1.2 \cdot c_{D}$. It can be seen from the table that the corrected design equations predict the experimental results well. This indicates that (17) is a good approximation of the displaced water inertia and the hydrodynamic propulsion mechanism is described more accurately.

TABLE III. $\quad U_{\text {VEHILLE }}$ (EXPERIMENT VS. THEORY, CORRECTED $I_{z z}^{a m}$ )

\begin{tabular}{|c|c|c|}
\hline$f[\mathrm{~Hz}]$ & $U_{\text {exp }}[\mathrm{m} / \mathrm{s}]$ & $U_{\text {theor }}[\mathrm{m} / \mathrm{s}]$ \\
\hline 0.5376 & 0.033 & 0.034 \\
\hline 0.8929 & 0.052 & 0.057 \\
\hline 1.1364 & 0.058 & 0.073 \\
\hline 1.3889 & 0.067 & 0.089 \\
\hline 1.7857 & 0.100 & 0.115 \\
\hline 2.0833 & 0.112 & 0.134 \\
\hline 2.5000 & 0.138 & 0.160 \\
\hline 3.1250 & 0.172 & 0.201 \\
\hline
\end{tabular}

\section{CONCLUSIONS}

This paper focused on the analysis, the design and the development of a small low-cost robotic fish propelled by an oscillating foil. A dynamic model for its linear motion was developed considering the hydrodynamic drag of the hull and the tail's propulsive thrust. Based on this model, the dimensions of the body and the tail were selected systematically. In addition, the same model was used to size the tail actuator, which is controlled by a $\mu \mathrm{C}$ running a $\mathrm{PD}$ controller. A wireless communication system and a power system were also implemented and all the subsystems were integrated in a hydrodynamic hull. The robotic fish was evaluated experimentally with respect to the tail motion system, the vehicle's hydrodynamic drag and the developed thrust. The experimental data lead to an improvement of the assumed tail model. The conclusions showed that the model of the hydrodynamic drag is sufficient provided that a better estimate for the moment of inertia of the water accelerated by the tail $I_{z z}^{a m}$ is used. Experiments showed that this inertia is significantly smaller than the one assumed originally.

\section{ACKNOWLEDGMENT}

The authors thank Prof. G. Triantafyllou for his suggestions in this research work, D. Tzeranis for initial work on the robotic fish, and M. Makrodimitris for supportive work. Support by the NTUA Senate Committee of Basic Research, Program "PEVE 07", is acknowledged.

\section{REFERENCES}

[1] M. S. Triantafyllou and G. S. Triantafyllou, An Efficient Swimming Machine, Scientific American, pp.40-48 (Mar. 1995).

[2] J. M. Anderson, K. Streitlien, D. S. Barrett and M. S. Triantafyllou, Oscillating Foils of High Propulsive Efficiency, J. Fluid Mechanics, vol. 360, pp.41-72 (1998).

[3] D. S. Barrett, M. S. Triantafyllou, D. K. P. Yue, M. A. Grosenbaugh and M. J. Wolfgang, Drag Reduction in Fish-like Locomotion, $J$. Fluid Mechanics, vol. 392, pp.183-212 (1999).

[4] J. M. Kumph and M. S. Triantafyllou, A Fast-Starting and Maneuvering Vehicle, the ROBOPIKE, Proc. of the Int. Symposium on Seawater Drug Reduction, Newport, RI, pp.485-490 (July 22-24, 1998).

[5] J. M. Anderson and P. A. Kerrebrock, The Vorticity Control Unmanned Undersea Vehicle (VCUUV) Performance Results, Proc. of the $11^{\text {th }}$ Int. Symp. on Unmanned Untethered Submersible Technology, Durham, NH, pp.360-369 (August 1999).

[6] M. Sfakiotakis, D. Lane and B. Davies, Review of Fish Swimming Modes for Aquatic Locomotion, IEEE J. of Oceanic Engineering, volume 24, No 2, pp.237-252 (April 1999).

[7] D. Read, F. Hover and M. S. Triantafyllou, Forces on Oscillating Foils for Propulsion and Maneuvering, J. of Fluids and Structures, vol. 17, pp.163-813 (2003).

[8] J. N. Newman, Marine Hydrodynamics, MIT Press, Cambridge, Massachusetts (1977).

[9] S. Hoerrner, Fluid-Dynamic Drag, published by the author (1965).

[10] D. Tzeranis, E. Papadopoulos and G. S. Triantafyllou, On the Design of an Autonomous Robot Fish, Proc. of the 11th IEEE Mediterranean Conf. on Control and Automation, Rhodes (June 17-20, 2003).

[11] E. Papadopoulos and G. Chasparis, Analysis and Model-based Control of Servomechanisms with Friction, Proc. 2002 Int. Conf. on Intelligent Robots and Systems, Lausanne, Switzerland, pp.21092114 (2002). 